\title{
EXPERIMENTAL INVESTIGATION OF THE EFFECTS OF NANOFLUIDS ON FORCED CONVECTIVE HEAT TRANSFER ALONG A MICROCHANNEL
}

\author{
Jonathan A Yeager1, Peter Daluga1, Lucas Splingaire1, Saeid Vafaei1* \\ 1Bradley University, Peoria, IL 61606, USA
}

\begin{abstract}
The demand for more compact and efficient heat exchangers has led to the use of microchannels, which allow for smaller, higher surface-to-volume ratio heat exchangers. To further increase efficiency, nanofluids-or fluids with nano-scale particles suspended in them-have become a high interest field of study. These nanofluids allow for more efficient heat exchangers by enhancing its heat transfer coefficient. This paper studies the effect that nanofluid has on forced convection heat transfer coefficient through a microchannel. The variation of heat transfer coefficient was experimentally measured for various nanofluids. Numerous stock nanoparticles were tested, as well as a custom-synthesized semiconductor $\mathrm{TiO}_{2}$ particle with anatase crystal phase. The data was then examined for the effect nanofluids had on forced convection heat transfer coefficient with respect to the dimensionless length, $x / D$. The experiment pushes the nanofluid through a stainless-steel microchannel with inner diameter of $0.31 \mathrm{~mm}$. Thermocouples were attached at the pipe inlet and outlet, as well as along its surface to obtain the thermal profile. The pipe and thermocouples were thermally insulated, and a DC current was used to generate a constant heat flux through the pipe. The input power, absorbed power, local heat transfer coefficient, and Nusselt number can be calculated through the gathered measurements. By using nanofluids, the developed heat transfer coefficient could be increased by over $143 \%$. It was also found that higher Reynolds numbers led to higher developed heat transfer coefficients in nanofluid trials; a $160 \%$ increase was observed for $\mathrm{TiO}_{2}$-water nanofluid at $\mathrm{Re}=400$ over $\mathrm{Re}=100$.
\end{abstract}

KEY WORDS: Forced Convection Heat Transfer, Nanofluid, Nanoparticles, Microchannel, Heat Transfer Coefficient, Nondimensionalized Length, Laminar Regime

\section{INTRODUCTION}

One region of the expanding edge of heat transfer technology is the development and expansion of the understanding of nanofluids, which consist of a solid nanoparticle suspended in a liquid solvent. This augmentation of working fluids has applications across numerous fields of science and engineering, including refrigeration, electronics, and solar collectors [1]. One application even used $\mathrm{TiO}_{2}$ and soluble oil as a cutting fluid [2]. Other common solutes are water and ethylene glycol. The solute properties play an important role in defining the final nanofluid characteristics.

There are numerous properties that can affect that nanofluid character. The size, shape, and concentration of the particle, the choice of material for both particle and base fluid, the presence of a surfactant, and the flow character all can weigh in, though these effects are not yet fully understood. While pipe size is more securely understood, fluid dynamics may become unpredictable as the diameter shrinks to the micro-scale. One method of making standard piping more analogous to micro-scale channels is the nondimensionalization of length, characterized as $\mathrm{x} / \mathrm{D}$, where $\mathrm{x}$ is the length along the pipe and $\mathrm{D}$ is the pipe diameter. There is a broad selection of potential nanoparticle materials, carrying with them an equally wide assortment of properties. A few commonly used metals are copper, titanium, and aluminum. Some studies also used silicon or carbon nanotubes. By substituting different nanoparticle materials, the overall character may drastically change. 
These benefits do not come without drawbacks. The improvements made to various key properties often come with increased pressure drops, requiring a higher input energy in pumping power for similar flows. Stability can also be a concern, as particles will agglomerate over time, sometimes requiring adding a surfactant or employing an agitator to counteract. Nanoparticles can be expensive to purchase and difficult to manufacture accurately, as stock nanoparticles may have issues with high variance in size and shape [3].

Much existing work has already been accomplished on the properties of nanofluids as a function of the base fluid and nanoparticle characteristics. The nanofluid density can be calculated through a simple calculation that applies to general mixtures, shown in Equation (1).

$$
\rho_{n f}=\phi \rho_{p}+(1-\phi) \rho_{b f}
$$

Likewise, the specific heat of the nanofluid, as shown in Equation (2), is a simple property calculation.

$$
\left(\rho c_{p}\right)_{n f}=\phi\left(\rho c_{p}\right)_{p}+(1-\phi)\left(\rho c_{p}\right)_{b f}
$$

Many correlations exist to predict nanofluid properties such as viscosity [4]. Duangthongsuk and Wongwises [5] developed an equation to calculate the viscosity of the nanofluid as a function of the base fluid viscosity and the nanoparticle concentration, as shown in Equation (3).

$$
\mu_{n f}=(1+2.5 \phi) \mu_{b f}
$$

Another innovation in heat transfer is the utilization of microchannels, which is a general term used for pipes under one millimeter in diameter. Due to the extremely small thickness and diameter of a microchannel, it has a very small thermal resistance, allowing the fluids in a heat exchanger application to be closer together, increasing the rate of heat transfer. While a length of microchannel has a very small cross-section, limiting flow and heat transfer rates, it is much smaller than a conventional pipe, allowing it to be packed much more densely. This is analogous to replacing one high-amperage resistor in a circuit with a large quantity of lowamperage resistors in parallel, each transmitting a small fraction of the overall current. This innovation is particularly useful in making heat exchangers smaller and lighter for applications such as satellites. One downside of the pipe size is that the individual pipes are very delicate, and special care may need to be taken to avoid damaging them.

\section{EXPERIMENTAL SETUP}

A diagram of the apparatus to determine heat transfer coefficient is shown in Figure 1. Fluid was pumped through the system by a New Era Pump Systems model NE-1000 syringe pump using a $100 \mathrm{~mL}$ Hamilton syringe (model 86020). The pump could be set to operate between $40 \mu \mathrm{L} / \mathrm{hr}$ and $2900 \mathrm{~mL} / \mathrm{hr}$, allowing for simple control of flow character and Reynolds number. The syringe pumped through a section of plastic tubing (Hamilton 86510) into a four-way junction (Upchurch Scientific 5700184).

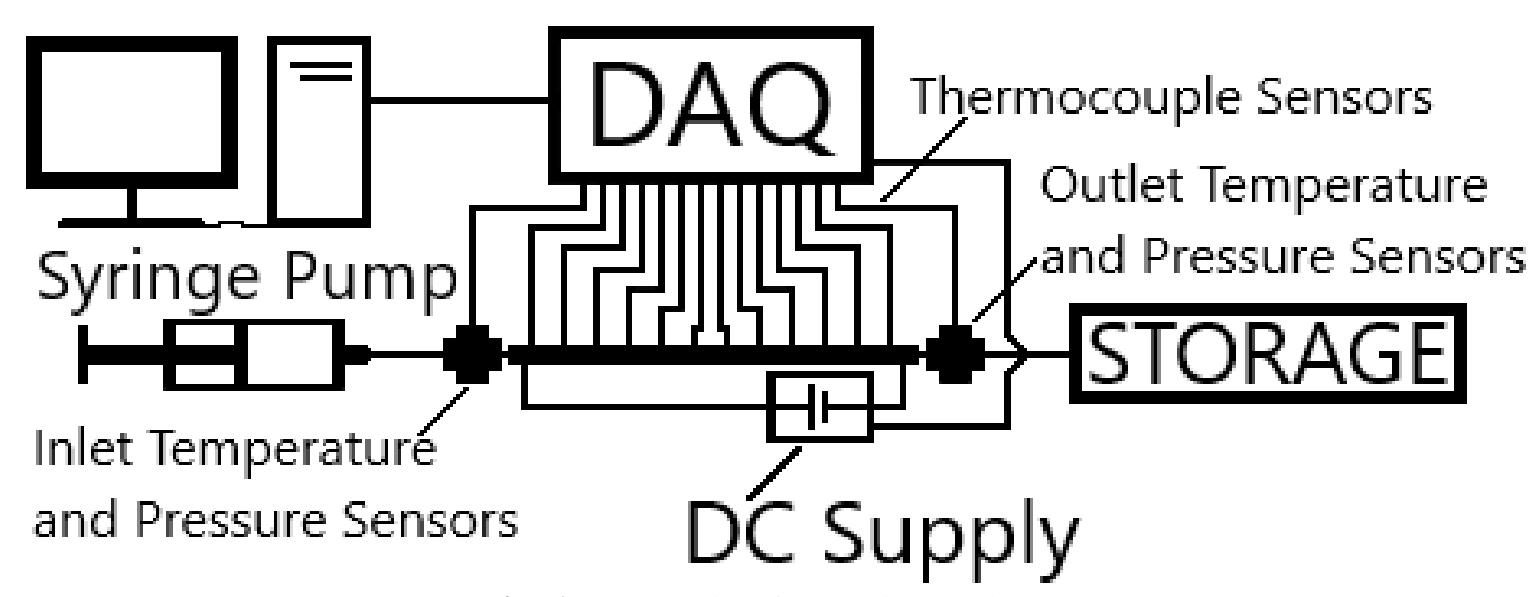

Fig. 1 Schematic of Experimental Setup 


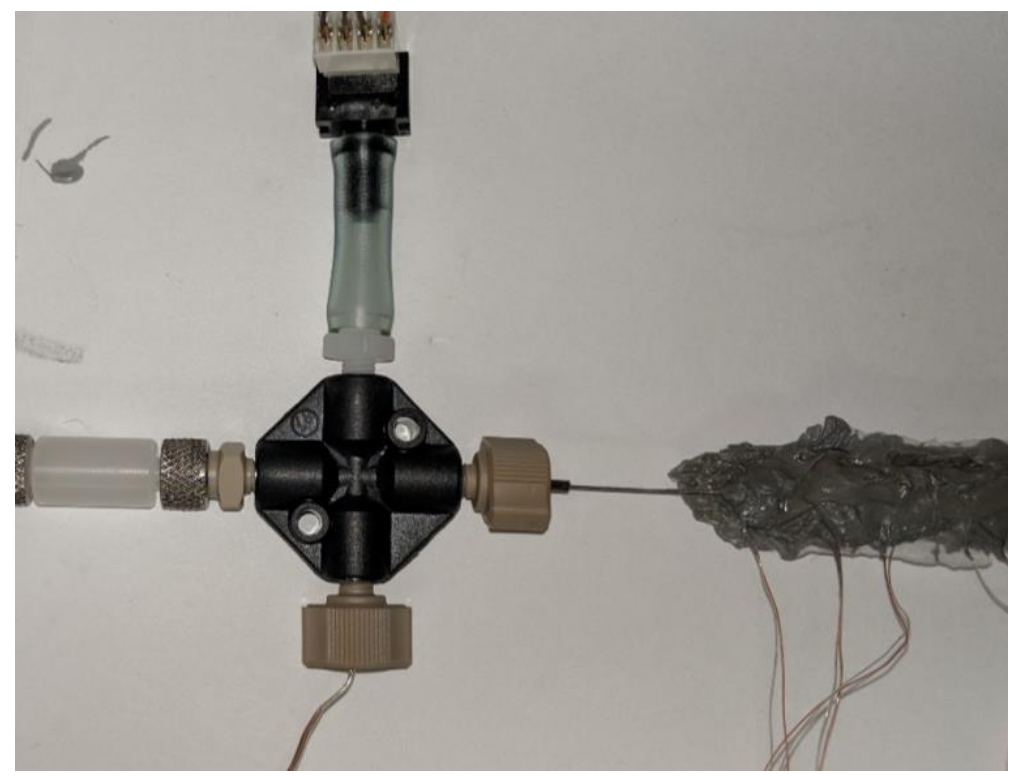

Fig. 2 Inlet Junction to the Microchannel with Attached Sensors

The hard-plastic junction provided attachment points (shown in Figure 2) for two sensors in addition to the flow system while dividing the fluid path into modular portions, providing ease of assembly. One of the sensors was an Omega PX26-100GV pressure transducer powered through an Omega PST 4130 regulated power supply set to output at $12 \mathrm{VDC}$ and $150 \mathrm{~mA}$. This reading was compared to the pressure after the microchannel to determine pressure drop. An Upchurch Scientific 5700142 adapter was used in addition to a section of stock tubing to allow the transducer to work with the junction. The other sensor was a $0.2 \mu \mathrm{m}$ thermocouple (OMEGA 5TC-TT-K-30-36), which was used to determine the net change of fluid temperature across the microchannel. This data was used with the flow rate to determine the rate of power absorption by the working fluid. There was a similar junction at the end of the microchannel, with a longer piece of plastic tubing leading to a spare bottle for waste collection and ease of transport. One difficulty caused by the small cross-section of the microchannel was a high pressure drop across it, which could have led to leaking from the junction. This was counteracted by using Loc-Tite epoxy to restrict the flow to the main path.

A section of rubber tube was necessary to help contain the flow out of the junction and into the microchannel, forming a seal when fully assembled. The microchannel itself was a 25-centimeter-long horizontal stainlesssteel needle (Hamilton 21027A) with inner and outer diameters of 210 and $413 \mu \mathrm{m}$, respectively. Due to the small size of the microchannel, special care had to be taken to avoid any forms of bending or crushing, which could render the system inoperable. One measure was keeping the system on an acrylic plate, assisting in transportation without directly contacting the pipe.

Along the pipe, 20 thermocouples (86- $\mu \mathrm{m}$ diameter, RS Pro 397-1589) were attached at centimeter intervals onto the pipe to obtain the temperature profile along the pipe surface (Figure 3). Spacing between thermocouples was made more precise by first marking the microchannel at the intervals, limiting placement error to less than 1 millimeter. The choice of 1 centimeter as a distance allowed for good resolution of the thermal development region along the pipe while leaving space between each for ease of assembly. An entry distance of $5 \mathrm{~cm}$ was left unheated to allow the fluid to fully develop hydraulically before developing thermally. The thermocouples were connected using a high thermal-conductivity epoxy glue (Cotronics Duralco 132). The glue helped secure the connection while also minimizing temperature difference between the pipe wall and thermocouple tip. A layer of 3M scotch-weld 2214 epoxy glue was also added as insulation and structural support over this section. In addition to this measure, a layer of dry insulation was added.

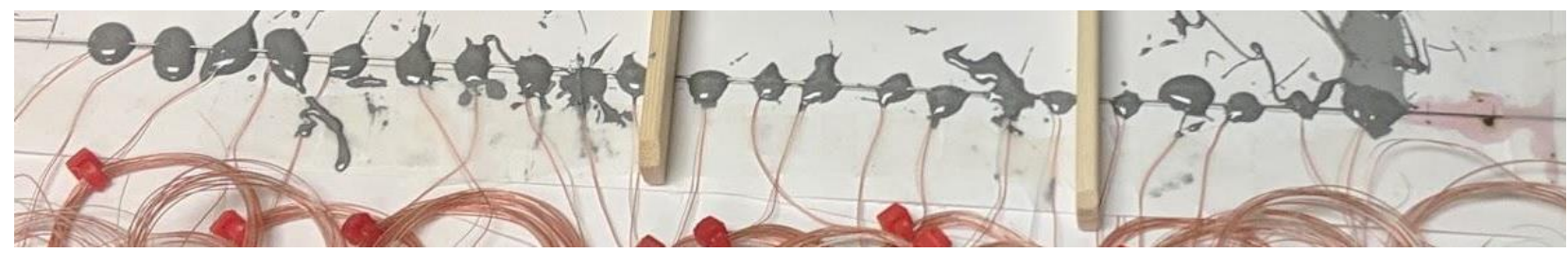

Fig. 3 The array of thermocouples glued to the microchannel with high-thermal-conductivity glue is shown. 


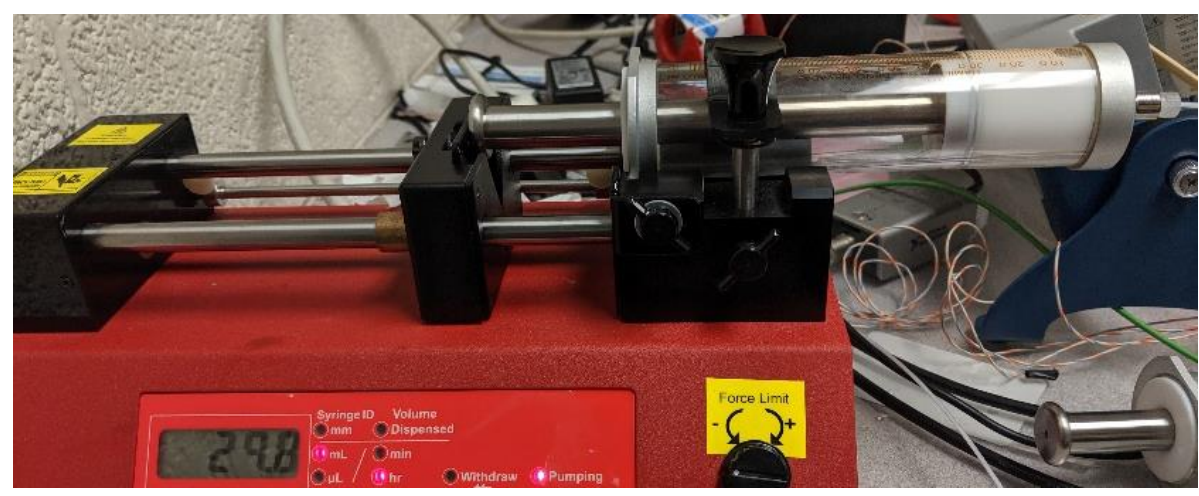

Fig. 4 A syringe is mounted into the syringe pump to allow nanofluid to flow through the system. The syringe is shown loaded with $\mathrm{TiO}_{2}$ nanofluid.

The test section was heated through electrical resistance by a Sorensen XPH 20-20 DC power supply. Sections of stock wire were connected to both ends of the testing region. The power supply was rated to 0-20 V and 0$20 \mathrm{~A}$, and displayed the current and voltage, which were used to calculate generated heat through the law of conservation of energy. The electrical resistance across the pipe was measured using a National Instruments USB-4065 Digital Multimeter attached across it. This real-time measurement allowed the experiment to account for variation with respect to temperature.

Thermocouples were attached to measurement and recording software (LabVIEW 2019) through a National Instruments NI 9213 card and a National Instruments NE cDAQ-9178 base. The power transducers fed into the same base through a National Instruments NI 9218 card with NI 9982 adapters. Voltage drop across the pipe was measured through a National Instruments NI 9221 card. Data was output to a spreadsheet for analysis. As measurements were performed while the system was at steady state, multiple measurements taken over a short time period could be averaged to lower fluctuation.

The experiment was conducted by first preparing the nanofluid. Due to the hazardous nature of working with nanoparticles, all manipulation with the powdered form was performed under a fume hood. In order to accurately obtain weight concentration an electric balance with an isolation box was used to eliminate disturbance from air currents. De-ionized water was measured in a similar fashion. The nanofluid was initially mixed with a glass stirrer, then transferred to a small jar and agitated with a reference shaker to assist in stably dispersing the nanoparticles. As one nanofluid trial was run, another would be queued up on the shaker to save time during testing.

Once the nanofluid was ready it was drawn into the syringe, which was then loaded onto the pump and connected to the input plastic tubing line, as shown in Figure 4. Flow was started at a low Reynolds number, ramping up to the desired flow condition. After flow was steady, the measurement system was enabled to ensure that fluid would remain below 80 Celsius, leaving a 20-degree safety barrier before the boiling point. As such, for testing with water, the inlet temperatures ranged from 20-25 Celsius, while the outlet temperatures ranged from 40 to 80 Celsius. As water was the only base fluid used in this experiment, and solubility generally increases with temperature, no other emergency temperatures were needed. Evaporating the fluid could cause catastrophic results to the experimental setup if nanoparticle were to deposit. The flow could become disrupted or even blocked, rendering the setup useless and requiring a reinvestment of time and capital into the experimental setup. Low weight concentrations were chosen for this experiment to help mitigate that risk.

After these preparations were made, testing commenced. The circuit was completed by connecting the current line to the DC power supply, which was pre-set to zero volts. The power was gradually increased to the desired heat flux, and a reading was taken once the system hit a steady state. Readings were taken over a period of 1020 seconds to have

After readings were taken, special care was taken to flush the system of nanofluid. Multiple fills of de-ionized water were used to clean the syringe, and the fluid system was run at a high flow rate for upwards of fifteen minutes to minimize deposition of any nanoparticles that may have stayed in the pipe. This was to avoid any deposition occurring along the insides of the pipe. 
Table 1

Summary of nanoparticles used in this study

\begin{tabular}{lllllll}
\hline Base Fluid & Nanoparticle Material & Source & Shape & Size & Weight $\%$ & Surfactant \\
\hline Water & ${\text { Anatase } \mathrm{TiO}_{2}}_{\text {Water }}^{\mathrm{Al}_{2} \mathrm{O}}$ & Custom 1 & Spherical & $20 \mathrm{~nm}$ & $1 \%$ & N/A \\
Water & Diamond Nanopowder & Stock US3008a & Spherical & $80 \mathrm{~nm}$ & $1 \%$ & N/A \\
\hline
\end{tabular}

1Particle synthesis process explained below

${ }_{2}$ Surfactant concentration $0.1 \%$

a US Research Nanomaterials, Inc

In this study, deionized water was used as a base fluid for all trials. The assortment of stock and custom nanoparticles used is shown in Table 1. Stock nanoparticles were from US Research Nanomaterials, consisting of $\mathrm{Al}_{2} \mathrm{O}_{3}$ (US3008) and diamond nanopowder (US1066), while the diamond nanopowder required use of a surfactant (US0010). Semiconductor anatase $\mathrm{TiO}_{2}$ nanoparticles designed for use in solar cells were also tested.

In addition to the stock nanoparticles, a semiconductor $\mathrm{TiO} 2$ nanoparticle with an anatase crystal phase was developed by the research group to investigate its potential merit to the field of nanofluid heat transfer. The procedure of nanoparticle production was as follows: In order to synthesize the $\mathrm{TiO}_{2}$ semiconductor nanoparticles, titanium chloride $\left(\mathrm{TiCl}_{4}\right)$ was mixed with a solution of sodium hydroxide $(\mathrm{NaOH})$, methanol $\left(\mathrm{CH}_{3} \mathrm{OH}\right)$, dimethylformamide (DMF), and de-ionized (DI) water. This mixture was then placed in a water bath at 40॰ C and stirred for 4 days (Figure 5). The mixture needs to be stirred to make sure crystallization would be homogeneous. After this, the mixture became white and gelatinous.

The mixture was then placed in centrifugation containers and centrifuged at $4000 \mathrm{rpm}$ for 10 minutes (Figure 6). This resulted in the gel being separated from the liquid. The liquid was discarded, and the gel was placed in tubes that were placed in liquid nitrogen for 5 minutes (Figure 7) to freeze the mixture of nanoparticles and base liquid. After removing the tubes from the liquid nitrogen, the caps are removed from the tubes, and the tubes are placed inside a jar can covered with a filter (Figure 8). This jar is attached to a dry-freeze apparatus, and a vacuum is pulled at roughly $26.67 \mathrm{kPa}$ for 24 hours (Figure 8).

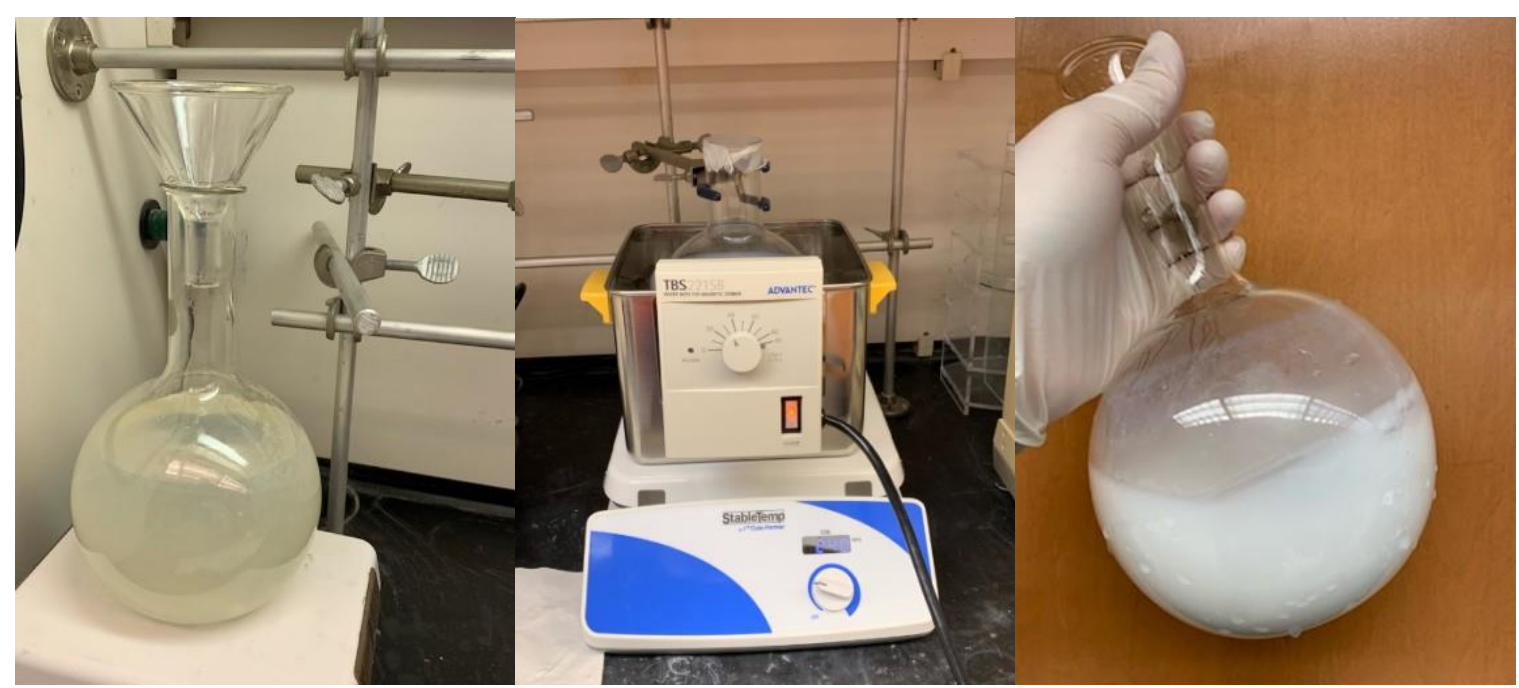

Fig. 5 The reaction mixture is prepared in a flask (left) and placed in a water bath (center). After 4 days of stirring, the mixture becomes white and gel-like (right). 
TFEC-2020-32141

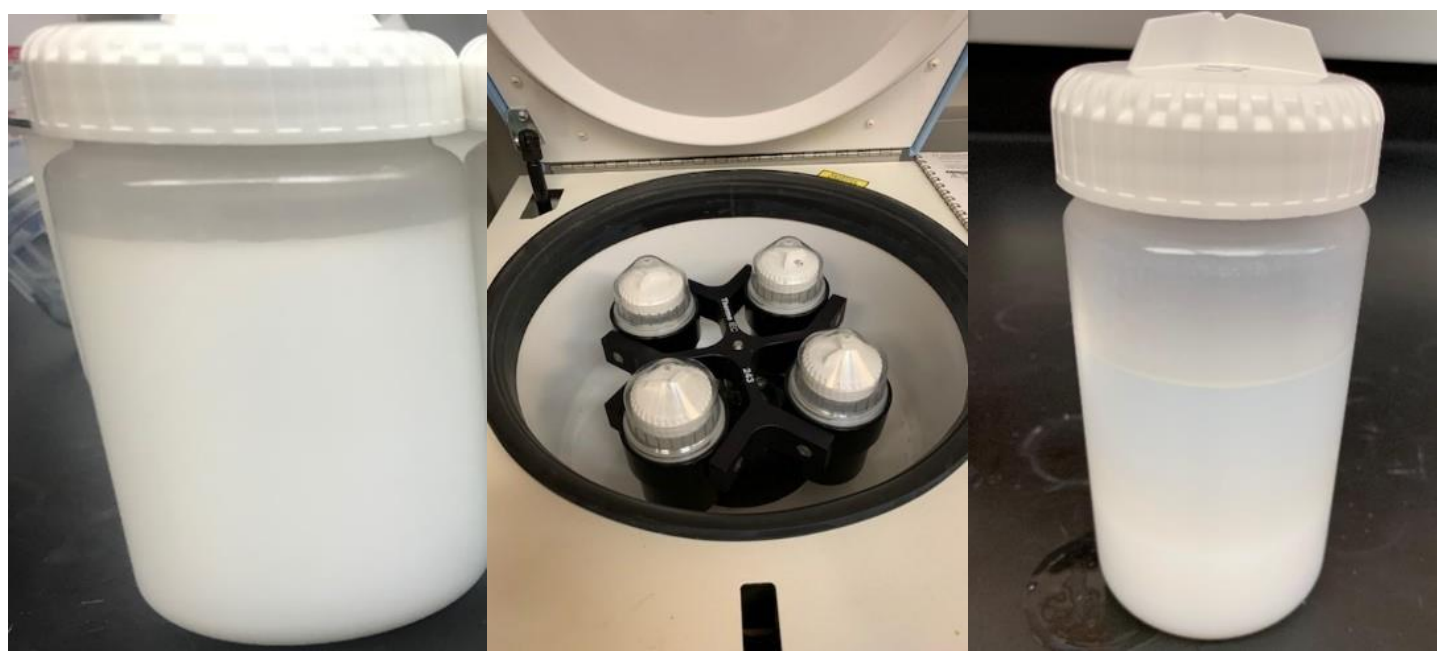

Fig. 6 The material is placed in centrifugation containers (left) and centrifuged (center). The result is the separation of the gel from the liquid (right).

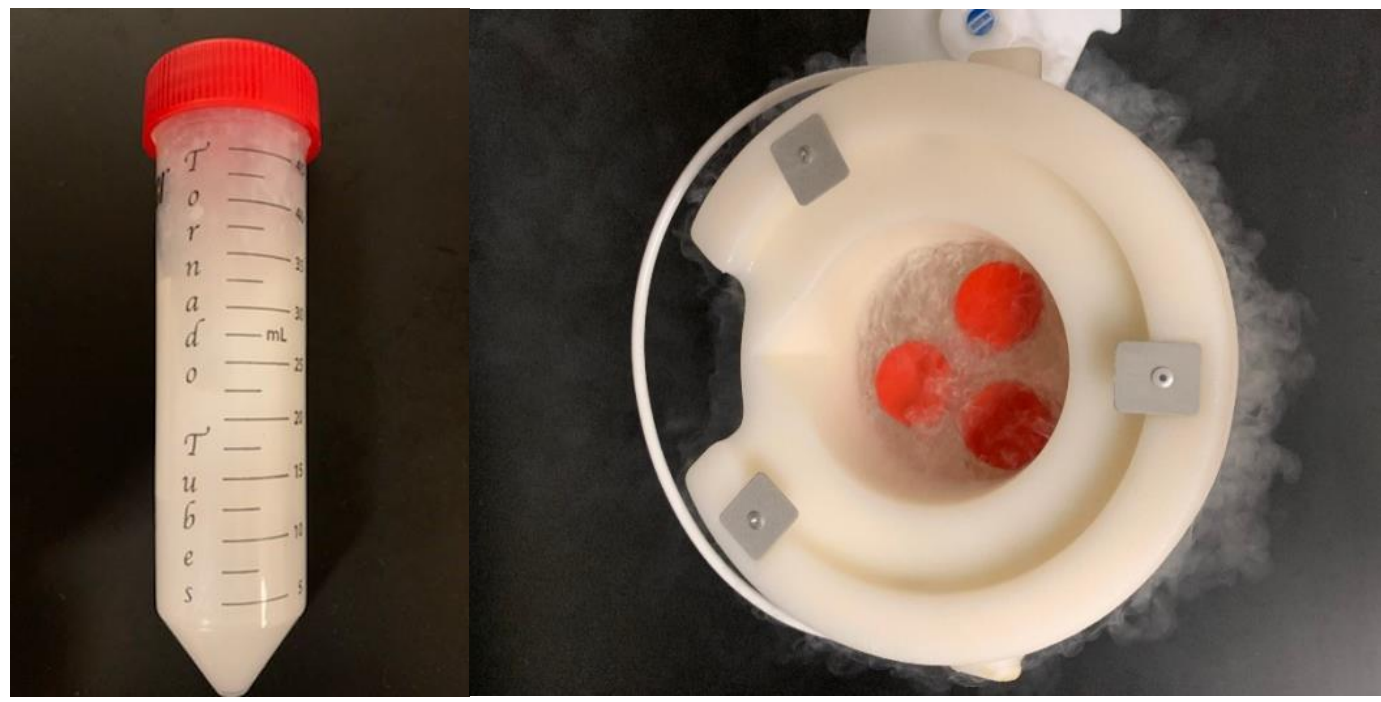

Fig. 7 The gel is placed in tubes (left) which are submerged in liquid nitrogen (right).

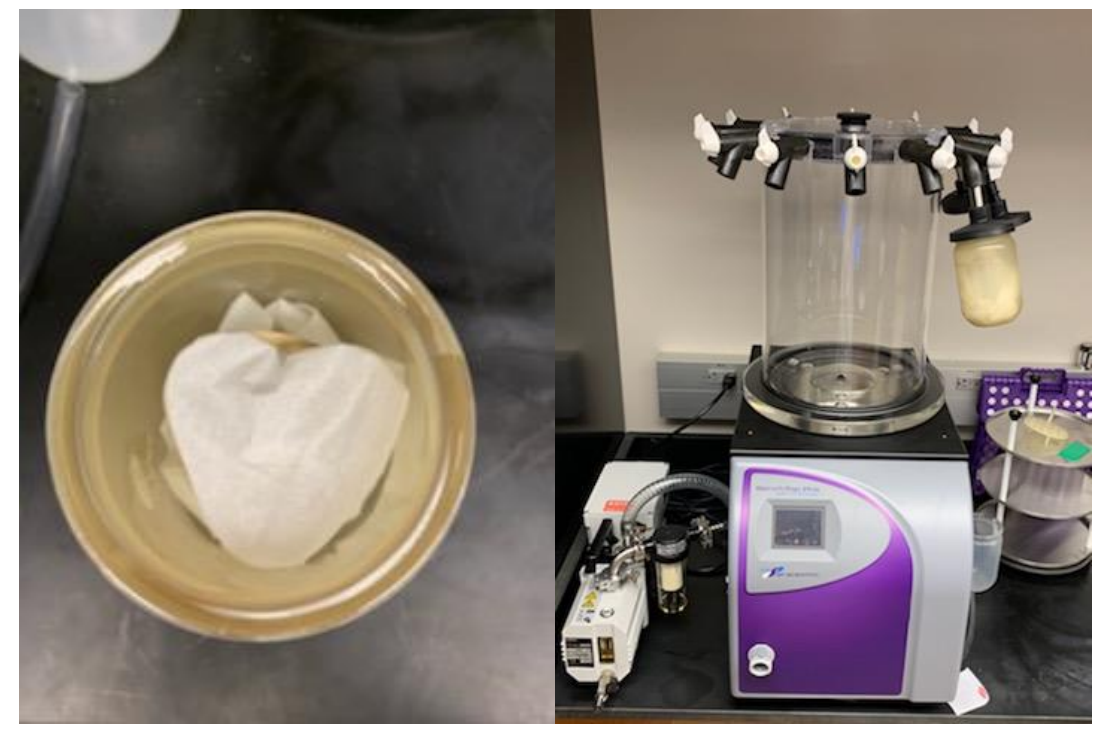

Fig. 8 The tubes are covered with a filter (left) and placed in the dry-freeze apparatus (right). 


\section{RESULTS AND DISCUSSION}

A series of calculations was required to determine the heat transfer coefficient profile along the pipe. Fluid properties calculated from Equations (1-3) are shown in table 2. Particle and base fluid properties were found at the average temperature between the inlet and outlet. Using the mass flow rate with the difference of inlet and outlet temperatures and the nanofluid specific heat, the rate of energy absorption by the working fluid ( $\left.\dot{Q}_{\text {absorbed }}\right)$ was found [Equation (4)].

$$
\dot{Q}_{\text {absorbed }}=\dot{m} c_{p} \Delta T=\dot{m} c_{p}\left(T_{\text {out }}-T_{\text {in }}\right)
$$

The rate of energy generation $\left(\dot{Q}_{g e n}\right)$ along the pipe was determined to be the power of the electric circuit element, the product of voltage and current [Equation (5)].

$$
\dot{Q}_{g e n}=I V
$$

The rate of heat loss to the surroundings $\left(\dot{Q}_{\text {loss }}\right)$ could then be found using conservation of energy [Equation (6)].

$$
\dot{Q}_{\text {loss }}=\dot{Q}_{g e n}-\dot{Q}_{\text {absorbed }}
$$

As power generation was constant along the length of the pipe, and at steady state conditions the fluid temperature at any given point was constant, the fluid temperature at any given point within the pipe could be determined to increase linearly with distance [Equation (7)].

$$
T(x)=T_{i n}+\frac{\dot{q} \pi D}{c_{p} \dot{m}} x
$$

The temperature differential between the fluid and the wall at any measured point was then found to be the difference between the measured wall temperature $\left(T_{\text {wall }}\right)$ and the calculated fluid temperature at that measurement point, and finally the heat transfer coefficient was found in terms of rate of heat absorption of the fluid, the temperature differential, and fundamental properties of the pipe [Equation (8)].

$$
h=\frac{\dot{Q}_{\text {absorbed }}}{A_{\text {wall }}} * \frac{1}{\Delta T}=\frac{\dot{Q}_{\text {absorbed }}}{\pi D_{i} L} * \frac{1}{T_{S}(x)-T_{f}(x)}
$$

\begin{tabular}{|c|c|c|c|}
\hline Material & Density $[\mathrm{kg} / \mathrm{m} 3]$ & Specific Heat [J/kg-k] & Dynamic Viscosity [mPa-s] \\
\hline \multicolumn{4}{|c|}{ Nanoparticles } \\
\hline $\mathrm{TiO}_{2}$ & 3900 & 697 & - \\
\hline $\mathrm{Al}_{2} \mathrm{O}_{3}$ & 3970 & 955 & - \\
\hline Diamond & 3150 & 520 & - \\
\hline \multicolumn{4}{|c|}{ Base Fluid } \\
\hline Water & 9970 & 4187 & 1.0518 \\
\hline \multicolumn{4}{|c|}{ Nanofluid } \\
\hline $\mathrm{TiO}_{2}$-Water & 1004.5 & 4183.2 & 1.0586 \\
\hline $\mathrm{Al}_{2} \mathrm{O}_{3}$-Water & 1004.5 & 4186 & 1.0585 \\
\hline Diamond-Water & 1003.9 & 4178.9 & 1.0602 \\
\hline
\end{tabular}

Table 2

Summary of nanofluid properties 


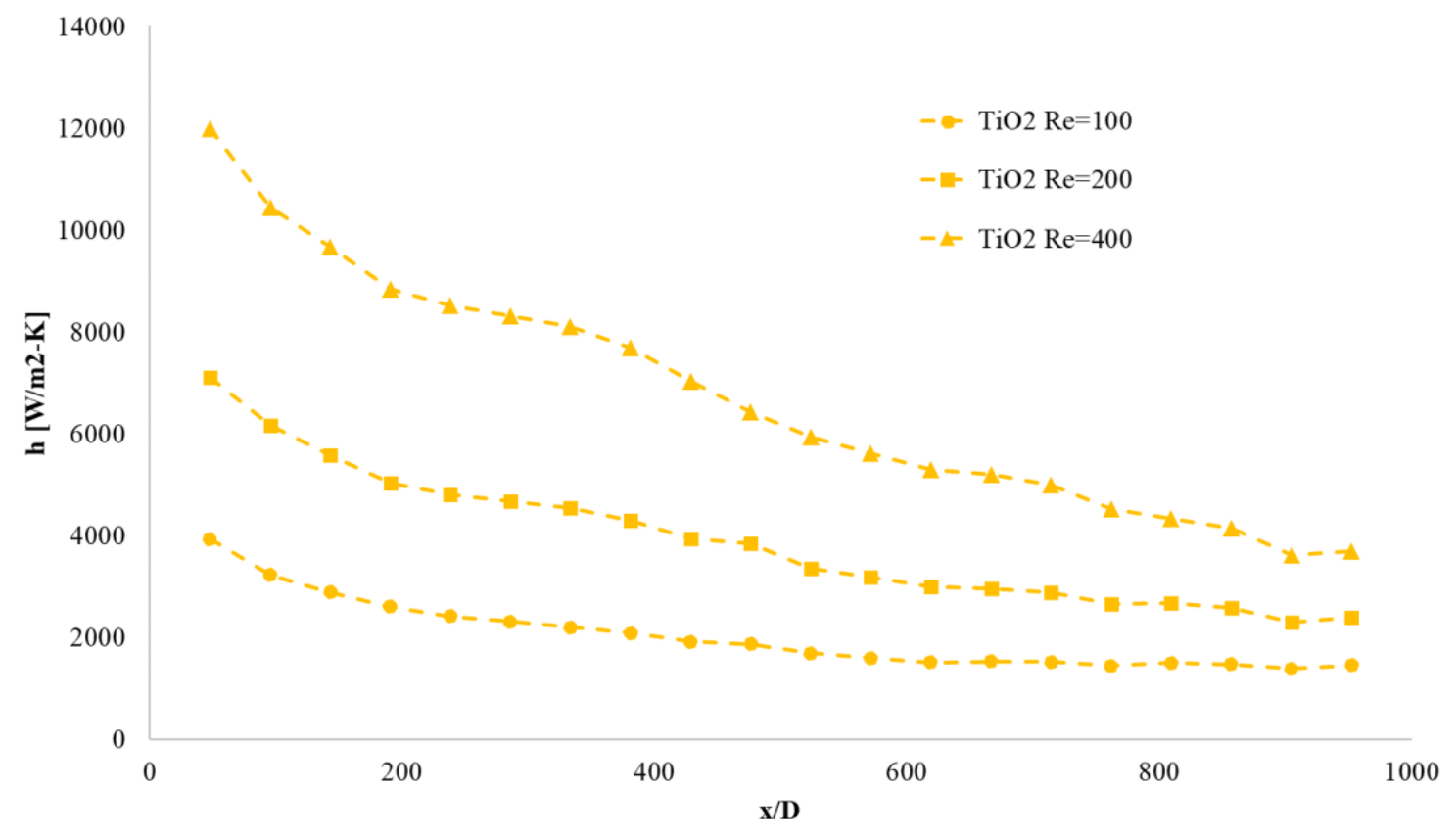

Fig. 9 Experimental trials for $\mathrm{TiO}_{2}$ at three different Reynolds numbers.

Figure 9 shows that as nondimensionalized length increased the flow developed thermally and the heat transfer coefficient decreased. This occurred as the internal flow heated up, decreasing the temperature differential between the wall and the fluid, which lowers the rate of heat transfer. Figure 9 also shows that heat transfer coefficient and thermal development length both increase with Reynolds number, which is supported by a multitude of correlations. The trial at $\mathrm{Re}=400$ saw the highest drop off, across the length, with a decrease of $70 \%$ across the thermal development region, while the trial at $\mathrm{Re}=200$ saw a drop of $68 \%$ and the trial at $\mathrm{Re}=100$ saw a drop of $60 \%$. It is possible that the trial at $\mathrm{Re}=400$ would continue to fall slightly, as the flow may not have finished developing thermally by the end of the measurement region.

Figure 10 shows that all nanofluids outperformed pure water in terms of developed heat transfer coefficient at $\mathrm{Re}=100$, demonstrating a clear enhancement due to the addition of the nanoparticle. $\mathrm{Al}_{2} \mathrm{O}_{3}$ expressed the highest effects, with a $143 \%$ increase of developed heat transfer coefficient, whereas $\mathrm{TiO}_{2}$ and Diamond saw increases of $34 \%$ and $25 \%$, respectively. It appears that pure water may have a higher heat transfer coefficient at the beginning of the thermal development region, but it quickly develops, falling below the nanofluids.

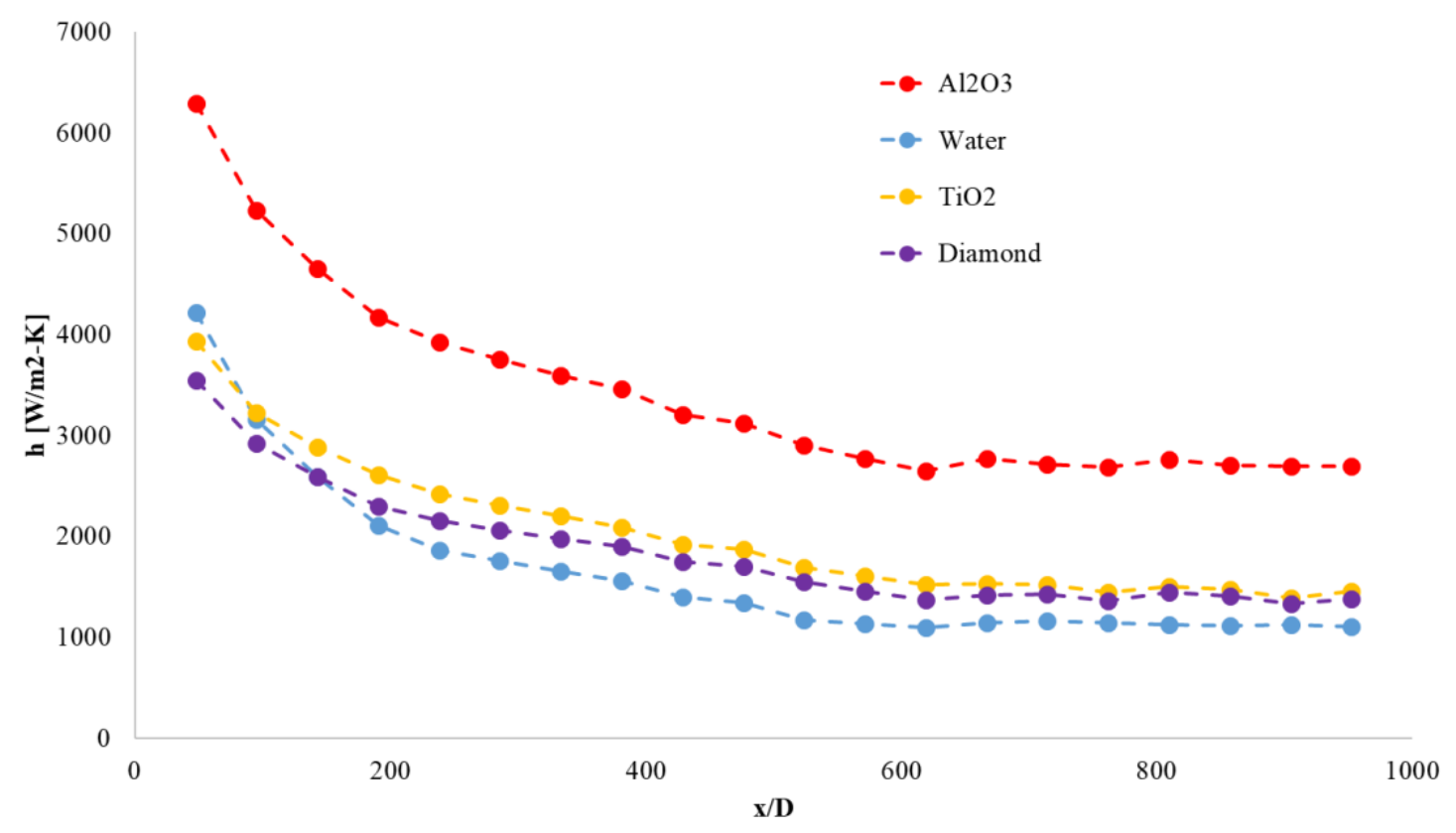

Fig. 10 Experimental trials for three nanofluids and pure water at $\operatorname{Re}=100$. 


\title{
CONCLUSIONS
}

An experiment was conducted to measure heat transfer coefficient along a microchannel as a function of nondimensionalized length. Multiple nanofluids were tested to see how they affected heat transfer. Working fluids were run through a microchannel by a syringe-pump based fluid system, and the pipe was heated through electrical resistance by a direct current. The thermal profile of the wall was measured by attaching a series of thermocouples at one-centimeter intervals over the length of the pipe. The gathered data affirmed that heat transfer coefficient increases with Reynolds number and decreases with nondimensionalized length. The developed heat transfer coefficient was shown to increase by $143 \%$ for an $\mathrm{Al}_{2} \mathrm{O}_{3}$-water nanofluid compared to pure water. This increase was much higher than that seen in other nanoparticle materials, demonstrating the importance of material choice. It was observed that heat transfer coefficient increased with Reynolds number for nanofluid trials, with $\mathrm{TiO}_{2}$-water nanofluid demonstrating a $160 \%$ increase at $\mathrm{Re}=400$ over $\mathrm{Re}=100$. The development region was also shown to increase in length at higher Reynolds numbers.

\section{ACKNOWLEDGEMENT}

This work was supported by the Illinois Space Grant Consortium, USA, and Bradley University.

\section{NOMENCLATURE}

$\begin{array}{lll}\text { I } & \text { Current } & \text { A } \\ \text { V } & \text { Voltage } & \text { V } \\ \varphi & \text { Volumetric Concentration } & -\end{array}$

\author{
Subscripts \\ bf Base fluid \\ $\mathrm{p}$ Particle \\ nf Nanofluid
}

\section{REFERENCES}

[1] Minea, A A., Moldoveanu, M., "Studies on A12O3, CuO, and TiO2 water-based nanofluids: A comparative approach in laminar and turbulent flow," Journal of Engineering Thermophysics, vol 26, pp 291-301, (2017)

[2] Salimi-Yasar et al., "Experimental investigation of thermal properties of cutting fluid using soluble oil-based $\mathrm{TiO}_{2}$ nanofluid," Powder Technology, vol 310, pp 213-220, (2017)

[3] Meriläinen et al., "Influence of particle size and shape on turbulent heat transfer characteristics and pressure losses in waterbased nanofluids," International Journal of Heat and Mass Transfer, vol 61, pp 439-448, (2013)

[4] Schlefhout, A, Vafaei, S., "Nanofluid Viscosity and Effective Parameters," 4th Thermal and Fluids Engineering Conference (TFEC), (2019)

[5] Duangthongsuk, Weerapun \& Wongwises, Somchai. "An experimental study on the heat transfer performance and pressure drop of TiO2-water nanofluids flowing under a turbulent flow regime," International Journal of Heat and Mass Transfer, vol 53, pp 334-344, (2010) 